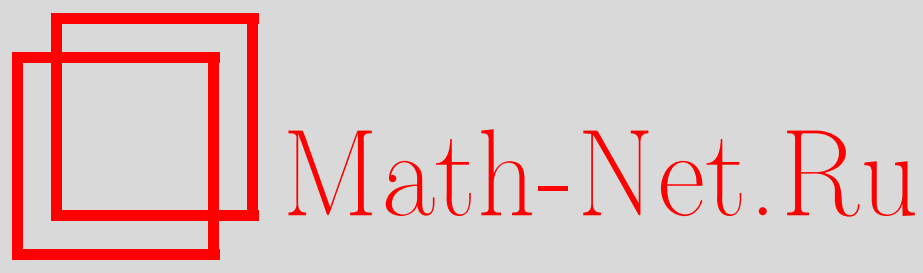

Г. Г. Козлов, Спектральная зависимость степени локализации в одномерной разупорядоченной модели Ллойда, TMФ, 2012, том 171, номер 1, 124-134

DOI: https://doi.org/10.4213/tmf6916

Использование Общероссийского математического портала Math-Net.Ru подразумевает, что вы прочитали и согласны с пользовательским соглашением http://www . mathnet.ru/rus/agreement

Параметры загрузки:

IP: 54.162 .127 .20

26 апреля 2023 г., 13:39:14

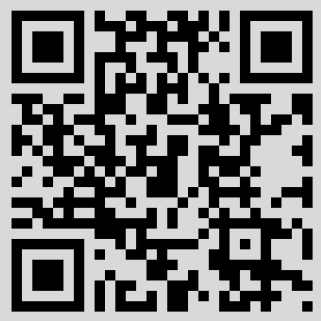




\title{
СПЕКТРАЛЬНАЯ ЗАВИСИМОСТЬ СТЕПЕНИ ЛОКАЛИЗАЦИИ В ОДНОМЕРНОЙ РАЗУПОРЯДОЧЕННОЙ МОДЕЛИ ЛЛОЙДА
}

\begin{abstract}
Для одномерных диагонально разупорядоченных моделей с функцией распределения узельных энергий, не имеющей конечных четных моментов выше нулевого, вычислены критерий Андерсона и спектральная зависимость степени локализации в первом неисчезающем приближении по беспорядку. Показано, что для этого класса моделей (когда обычная теория возмущений оказывается неприменимой) может быть последовательно построена теория возмущений для совместной статистики опережающей и запаздывающей функций Грина. Расчеты для модели Ллойда показали, что критерий Андерсона является в этом случае линейной (а не квадратичной, как обычно) функцией степени разупорядочения. Расчеты иллюстрируются компьютерными экспериментами.
\end{abstract}

Ключевые слова: андерсоновская локализация, одномерная разупорядоченная система, функция Грина.

\section{1. ВВЕДЕНИЕ, ПОСТАНОВКА ЗАДАЧИ И ОСНОВНЫЕ РЕЗУЛЬТАТЫ}

Одной из главных причин изучения одномерных $(1 D)$ систем в физике твердого тела является относительная простота соответствующих математических моделей. Это позволяет при анализе $1 D$-моделей в ряде случаев получать результаты в аналитическом виде и использовать их как наводящие при изучении более реалистичных двух- и трехмерных твердотельных моделей. В настоящее время, однако, есть основания полагать, что значение $1 D$-моделей этим не исчерпывается. Современные технологии производства материалов и методики экспериментов позволяют создавать и изучать низкоразмерные системы (например, квантовые сверхрешетки, $1 D$-фотонные кристаллы, оптические волокна), для анализа которых одномерные математические модели могут быть непосредственно использованы. Кроме того, в последнее время увеличивается интерес к известным естественным $1 D$-системам таким, как $J$-агрегаты [1], [2], спектроскопические свойства которых описываются в терминах $1 D$-экситона Френкеля.

* Научно-исследовательский институт физики им. В. А. Фока, Санкт-Петербургский государственный университет, Санкт-Петербург, Россия.

E-mail: gkozlov@photonics.phys.spbu.ru 
Неизбежные флуктуации технологических режимов при изготовлении "искусственных" $1 D$-систем и флуктуации окружения естественных одномерных систем порождают беспорядок, который необходимо учитывать при их описании. Математические модели беспорядка в одномерных системах составляют важную часть теории разупорядоченных систем [3] и продолжают исследоваться в настоящее время [4]-[6]. Об одном классе таких моделей пойдет речь в предлагаемой статье. Матрица гамильтониана Н моделей этого класса имеет стандартный вид ${ }^{1)}$

$$
H_{r r^{\prime}}=\delta_{r r^{\prime}} \varepsilon_{r}+\delta_{r, r^{\prime}+1}+\delta_{r, r^{\prime}-1}, \quad r, r^{\prime}=1,2, \ldots, N, \quad N \rightarrow \infty,
$$

а специфика заключается в том, что функция распределения $P(\varepsilon)$ случайных узельных энергий $\varepsilon_{r}$ может убывать настолько медленно, что все ее четные моменты, кроме нулевого, расходятся. Примером подобной модели может служить модель Ллойда [7], в которой $P(\varepsilon)$ является распределением Коши. Как известно, для модели Ллойда возможно точное вычисление усредненной функции Грина [3], [7], однако несмотря на то, что локализация собственных состояний в такой модели активно исследовалась [6], [8]-[12], полученные в нашей работе результаты, касающиеся спектральной зависимости степени локализации в смысле критерия Андерсона, по нашим сведениям, нигде не освещались. Отметим, что изучение подобных моделей представляет методический интерес, поскольку использование обычной теории возмущений в данном случае оказывается невозможным.

Для анализа локализации в моделях описанного выше класса мы применяем методику [13], основанную на построении совместной статистики опережающей и запаздывающей функций Грина. Целью настоящей работы является обобщение этой методики на случай диагонально разупорядоченных $1 D$-моделей описанного выше класса, когда формулы нашей работы [13], выражающие критерий Андерсона и спектральную зависимость локализации через второй момент функции распределения узельных энергий $\int P(\varepsilon) \varepsilon^{2} d \varepsilon$, оказываются неприменимыми. В качестве примера мы приведем расчеты для модели Ллойда и покажем, что зависимость критерия Андерсона от параметра ширины распределения узельных энергий в этом случае линейная, а не квадратичная, как для быстроубывающих распределений [13].

Общая постановка задачи и количественные определения вычисляемых ниже величин в точности соответствуют таковым из работы [13], поэтому здесь мы ограничимся лишь кратким напоминанием. Рассмотрим длинную разупорядоченную цепочку, описываемую гамильтонианом (1), и будем считать, что в начальный момент времени $t=0$ возбуждение было сосредоточено на крайнем узле $N$ этой цепочки. Введем критерий Андерсона $D$ как вероятность того, что возбуждение останется на исходном узле $N$ при $t \rightarrow \infty$. Как известно, в упорядоченной системе $D=0$, и появление (вследствии беспорядка) в спектре гамильтониана (1) локализованных состояний соответствует условию $D \neq 0$ [3], [13], [14].

Если $\Psi^{\lambda}$ и $E_{\lambda}$ суть собственные векторы и собственные числа матрицы (1), то величина $D$ может быть представлена в виде [13]

$$
D=\int W(U) d U, \quad \text { где } W(U) d U=\left\langle\sum_{E_{\lambda} \in[U, U+d U]}\left|\Psi_{N}^{\lambda}\right|^{4}\right\rangle .
$$

1) Гамильтониан такого вида описывает экситон Френкеля в одномерной диагонально разупорядоченной цепочке с взаимодействием только между ближайшими соседями. 
Скобки $\langle\cdot\rangle$ показывают усреднение по случайным узельным энергиям $\varepsilon_{r}$. Отличие функции $W(U)$ от нуля при какой-либо энергии $U$ свидетельствует о том, что собственные векторы (1) с энергией $U$ локализованы в смысле критерия Андерсона, т. е. эту функцию можно назвать спектральной зависимостью степени локализа$u$ u $u^{2)}$.

Как и в работе [13], функцию распределения энергий узлов $P(\varepsilon)$ мы представим в виде

$$
P(\varepsilon)=\frac{1}{\Delta} p\left(\frac{\varepsilon}{\Delta}\right), \quad p(x)>0, \quad \int p(x) d x=1,
$$

причем $\Delta$ является мерой разупорядочения системы, так как при $\Delta \rightarrow 0$ система становится упорядоченной. В работе [13] были получены следующие выражения для $D$ и $W(U): D=M_{2} \Delta^{2} / 2$ и $W(U)=M_{2} \Delta^{2} \sqrt{\left(4-U^{2}\right)} /(4 \pi),|U|<2$, где $M_{2}=\int p(x) x^{2} d x$. При $M_{2}<\infty$ эти выражения применимы в случае слаборазупорядоченных систем, когда $D \ll 1$. Основным результатом настоящей работы являются следующие применимые при $D \ll 1$ выражения для функции $W(U)$ и величины $D$ :

$$
W(U)=\frac{\left(4-U^{2}\right)^{3 / 2}}{4 \pi} \int d \varepsilon P(\varepsilon) \ln \left(1+\frac{\varepsilon^{2}}{4-U^{2}}\right), \quad|U|<2, \quad D=\int_{-2}^{2} d U W(U) .
$$

Эти выражения справедливы фактически при любой мыслимой функции $P(\varepsilon)$. При $M_{2}<\infty$ формулы (4) переходят (при малых $\Delta$ ) в формулы работы [13]. При $M_{2}=\infty$ зависимость $D$ от $\Delta$ качественно меняется. В частности, для модели Ллойда, когда $p(x)=\left[\pi\left(1+x^{2}\right)\right]^{-1}$, величина $D$ оказывается пропорциональной первой степени $\Delta$, что подтверждается описанным в заключительной части статьи компьютерным экспериментом.

\section{PACपET}

Для решения задачи, поставленной в предыдущем разделе, мы будем использовать предложенную в работе [13] методику, которую сейчас кратко опишем. Вероятность возбуждения $\left|\Psi_{N}(t)\right|^{2}$ на краевом узле $N$ разупорядоченной цепочки выражается через две краевые функции Грина $(\mathrm{K} \Phi \Gamma) \gamma\left(U_{1}\right)$ и $\gamma\left(U_{2}\right)$ при разных энергиях $U_{1}$ и $U_{2}$. Указанные КФГ являются случайными величинами, причем их совместная функция распределения $\rho\left(x_{1} x_{2}\right)$ удовлетворяет уравнению [13]

$$
x_{1}^{2} x_{2}^{2} \rho\left(x_{1} x_{2}\right)=\int P(\varepsilon) \rho\left(U_{1}-\frac{1}{x_{1}}-\varepsilon, U_{2}-\frac{1}{x_{2}}-\varepsilon\right) d \varepsilon .
$$

Искомая вероятность возбуждения на краевом узле $\left|\Psi_{N}(t)\right|^{2}$ связана с функцией $\rho\left(x_{1} x_{2}\right)=\rho_{U_{1} U_{2}}\left(x_{1} x_{2}\right)$ следующим образом [13]:

$$
\left|\Psi_{N}(t)\right|^{2}=\frac{i}{\pi^{2}} \int d U d \omega e^{-i \omega t}\left\langle y_{2} x_{1}\right\rangle
$$

где

$$
\left\langle y_{2} x_{1}\right\rangle=\pi \lim _{a \rightarrow \infty} a^{2} \int x \rho_{U, U+\omega}(x, a) d x .
$$

${ }^{2)} \mathrm{B}$ работе [13] $W(U)$ названа функцией участия, поскольку $W(U)$ имеет много общего с IPR (inverse participation ratio) [4]. 
При малых $\omega$ функция $\rho_{U, U+\omega}\left(x_{1}, x_{2}\right)$ имеет сингулярность типа полюса, т. е. представима в виде

$$
\rho_{U, U+\omega}\left(x_{1} x_{2}\right) \approx \operatorname{sing} \rho_{U, U+\omega}\left(x_{1} x_{2}\right)=\frac{1}{\omega} \mathcal{F}_{U}\left(x_{1} x_{2}\right) .
$$

Подставляя представление (7) в выражение (6) и учитывая, что $\int d \omega e^{-i \omega t} / \omega=-i \pi$, получаем, что предельная при $t \rightarrow \infty$ вероятность возбуждения на крайнем узле $D(2)$ определяется формулой

$$
D=\left|\Psi_{N}(t=\infty)\right|^{2}=\int W(U) d U, \quad \text { где } \quad W(U)=\lim _{a \rightarrow \infty} a^{2} \int d x \mathcal{F}_{U}(x, a) x,
$$

причем, как показано в работе [13], функция $W(U)$ в этом выражении имеет смысл спектральной зависимости степени локализации (2). Таким образом, для вычисления $D$ и $W(U)$ следует найти сингулярную по разности энергий $\omega$ часть $(7)$ решения уравнения (5). Эти сведения из статьи [13] являются исходными для настоящей работы. Ниже описывается схема расчета величин $D$ и $W(U)$ в первом неисчезающем приближении по степени разупорядочения $\Delta(3)$, пригодная для фактически произвольной осмысленной функции $p(x)(3)$, в том числе и тогда, когда все четные (кроме нулевого) моменты $p(x)$ расходятся. Как и в работе [13], все расчеть относятся $\kappa$ области энергий $|U|<2$, где плотность состояний упорядоченной модели отлична от нуля и находится подавляющее большинство состояний и в случае слабого беспорядка, который мы рассматриваем.

Нам будет удобно ввести функцию $\mathcal{R}\left(x_{1} x_{2}\right) \equiv \rho\left(U_{1}-x_{1}, U_{2}-x_{2}\right)$. С помощью уравнения (5) нетрудно убедиться в том, что $\mathcal{R}\left(x_{1} x_{2}\right)$ удовлетворяет следующему уравнению:

$$
\frac{\mathcal{R}\left(U_{1}-1 / x_{1}, U_{2}-1 / x_{2}\right)}{x_{1}^{2} x_{2}^{2}} \equiv \mathcal{H}_{U_{1}}\left(x_{1}\right) \mathcal{H}_{U_{2}}\left(x_{2}\right) \mathcal{R}\left(x_{1} x_{2}\right)=\int P(\varepsilon) \mathcal{R}\left(x_{1}+\varepsilon, x_{2}+\varepsilon\right) d \varepsilon
$$

В левой части этого уравнения фигурирует функциональный оператор $\mathcal{H}_{U}(x)$, который действует на произвольную функцию $f(x)$ следующим образом: $\mathcal{H}_{U}(x) f(x) \equiv$ $f(U-1 / x) / x^{2}$. Используем описанную в работе [15] систему собственных функций оператора $\mathcal{H}_{U}(x)$ для того, чтобы записать уравнение $(9)$ в матричном виде. Приведем полученные в работе [15] явные выражения для собственных функций $\sigma_{U}^{n}(x)$ и собственных значений $\lambda_{n}$ этого оператора при $|U|<2$ :

$$
\sigma_{U}^{n}(x)=\mathcal{L}_{U}(x)\left[\frac{R_{U}^{*}-x}{R_{U}-x}\right]^{n} \equiv \mathcal{L}_{U}(x) G_{U}^{n}(x), \quad \lambda_{n}=\left(\frac{U+i \sqrt{4-U^{2}}}{U-i \sqrt{4-U^{2}}}\right)^{n}, \quad\left|\lambda_{n}\right|=1,
$$

где

$$
G_{U}(x) \equiv \frac{R_{U}^{*}-x}{R_{U}-x}, \quad R_{U} \equiv \frac{U+i \sqrt{4-U^{2}}}{2}, \quad \mathcal{L}_{U}(x) \equiv \frac{1}{2 \pi i}\left[\frac{1}{x-R_{U}}-\frac{1}{x-R_{U}^{*}}\right] .
$$

Нам понадобится также полученное в работе [15] правило проецирования на систему функций (10), которое заключается в том, что произвольная функция $f(x)$ может быть представлена в виде ряда

$$
f(x)=\sum_{n=-\infty}^{+\infty} A_{n} \sigma_{U}^{n}(x), \quad \text { где } \quad A_{n}=\int \frac{f(x)}{G_{U}^{n}(x)} d x .
$$


С помощью правила (12) разложим правую и левую части уравнения (9) по системе функций (10):

$$
\begin{gathered}
\mathcal{R}\left(x_{1} x_{2}\right)=\sum_{n m} C_{n m} \sigma_{U_{1}}^{n}\left(x_{1}\right) \sigma_{U_{2}}^{m}\left(x_{2}\right) \\
\int P(\varepsilon) \mathcal{R}\left(x_{1}+\varepsilon, x_{2}+\varepsilon\right) d \varepsilon=\sum_{q p} \sum_{n m} \sigma_{U_{1}}^{q}\left(x_{1}\right) \sigma_{U_{2}}^{p}\left(x_{2}\right) J_{q p}^{n m} C_{n m}
\end{gathered}
$$

где

$$
J_{q p}^{n m} \equiv \int P(\varepsilon) \frac{\sigma_{U_{1}}^{n}\left(\varepsilon+x_{1}\right) \sigma_{U_{2}}^{m}\left(\varepsilon+x_{2}\right)}{G_{U_{1}}^{q}\left(x_{1}\right) G_{U_{2}}^{p}\left(x_{2}\right)} d x_{1} d x_{2} d \varepsilon .
$$

Приравнивая коэффициенты при функциях $\sigma_{U_{1}}^{n}\left(x_{1}\right) \sigma_{U_{2}}^{m}\left(x_{2}\right)$ в правой и левой частях уравнения (9), получим

$$
C_{q p} \lambda_{q}\left(U_{1}\right) \lambda_{p}\left(U_{2}\right)=\sum_{n, m} J_{q p}^{n m} C_{n m}
$$

Пронумеруем пары индексов одним индексом $q p \rightarrow \alpha$, причем паре индексов $q=p=0$ сопоставим $\alpha=1$. Тогда, если обозначить $\lambda_{q}\left(U_{1}\right) \lambda_{p}\left(U_{2}\right) \equiv \Lambda_{\alpha}$, то уравнение $(14)$ для всех $\alpha$ запишется в виде

$$
\sum_{\beta} M_{\alpha \beta} C_{\beta}=0, \quad M_{\alpha \beta}=J_{\alpha}^{\beta}-\Lambda_{\alpha} \delta_{\alpha \beta}
$$

Уравнение (15) и представляет собой искомый матричный эквивалент уравнения (9).

Решение уравнения (5) имеет смысл функции распределения и поэтому должно быть знакопостоянным. Аргументы, свидетельствующие о том, что любое решение (5) имеет определенный знак, приведены в приложении. Покажем, что из этого следует пропорциональность всех решений (5) друг другу, т. е. одномерность многообразия решений уравнения (5). Действительно, допустим, что существуют два линейно независимых решения (5) $\rho_{1}$ и $\rho_{2}$. Без потери общности мы можем считать их положительными. Но тогда решением будет и произвольная линейная комбинация $\rho=\rho_{1} c_{1}+\rho_{2} c_{2}$, причем коэффициенты $c_{1}$ и $c_{2}$ можно выбрать разного знака, так что при некоторых значениях аргументов $x_{1}$ и $x_{2}$ функция $\rho$ будет менять знак. Это противоречит полученному в приложении свойству знакопостоянства решений (5) и, следовательно, двух (и более) линейно независимъх решений у уравнения (5) (и связанных с ним уравнений (9) и (15)) быть не может.

Для матричного уравнения (15) это утверждение соответствует тому, что у особой матрицы $M$ (т. е. $\operatorname{det} M=0)$ все строки, кроме одной, являются линейно независимыми. Пусть линейно зависимой является строка матричного уравнения (15), соответствующая $\alpha=1$. Поскольку $\int \sigma_{U}^{n}(x) d x=\delta_{n 0}$ [15], то условие нормировки $\int d x_{1} d x_{2} \mathcal{R}\left(x_{1} x_{2}\right)=1$ соответствует требованию $C_{00}=1 \rightarrow C_{1}=1$. Если теперь обозначить через $L$ размер матрицы $M$ (разумеется, при вычислениях следует считать $L \rightarrow \infty)$, то нетрудно убедиться в том, что нормированное решение уравнения (15) может быть записано в виде

$$
\begin{aligned}
C_{\alpha} & =K(\Delta) e_{\alpha, i_{2}, i_{3}, \ldots, i_{L}} M_{2 i_{2}} M_{3 i_{3}} M_{4 i_{4}} \ldots M_{L i_{L}}, & & L \rightarrow \infty, \\
K(\Delta) & =\left[e_{1, i_{2}, i_{3}, \ldots, i_{L}} M_{2 i_{2}} M_{3 i_{3}} M_{4 i_{4}} \ldots M_{L i_{L}}\right]^{-1}, & & L \rightarrow \infty,
\end{aligned}
$$


где по повторяющимся индексам подразумевается суммирование от 1 до $L$, а $e_{i k l m . . . n}-$ символ Леви-Чивиты (полностью антисимметричная функция значков) $)^{3)}$. Напомним, что, во-первых, $e_{123 . . L}=1$ и, во-вторых, перестановка двух любых значков приводит к изменению знака (например, $e_{21345 \ldots L}=-1$ ). Следовательно, $e_{i k l m \ldots n} \neq 0$, только если среди значков $i k l m . . n$ нет совпадающих (например, $\left.e_{11345 \ldots L}=0\right)$.

Допустим, что мы имеем дело с упорядоченной системой, т. е. $\Delta=0$ и $P(\varepsilon)=\delta(\varepsilon)$. Тогда $J_{\alpha}^{\beta}(\Delta=0)=\delta_{\alpha \beta}$ и, следовательно, $M_{\alpha \beta}(\Delta=0)=\delta_{\alpha \beta}\left(1-\Lambda_{\alpha}\right)$. Из формулы (16) получаем, что $C_{\alpha}(\Delta=0)=\delta_{\alpha 1}$ (этот результат совпадает с результатом работы [13]) и

$$
K(0)=\prod_{\alpha=1}^{L}\left(1-\Lambda_{\alpha}\right)^{-1}, \quad L \rightarrow \infty .
$$

При переходе к разупорядоченной системе у матрицы $M$ появляются недиагональные элементы, малые по беспорядку $\Delta$ в том смысле, что они обращаются в нуль при $\Delta \rightarrow 0$. Поэтому для получения первого неисчезающего приближения для коэффициентов $C_{\alpha}$ в произведениях матричных элементов, входящих в формулу (16), следует оставить только один какой-либо недигональный элемент, а остальные (дигональные) считать невозмущенными. Что касается нормировочного коэффициента $K(\Delta)$, то при вычислении первой неисчезающей поправки его следует положить равным $K(0)(17)$. При таком подходе характер малости (цело-степенной, дробно-степенной или какой-либо другой) недиагональных элементов матрицы $M$ как функций $\Delta$ не играет роли. Таким образом, в первом неисчезающем приближении (мы показываем это приближенными равенствами) для коэффициентов $C_{\alpha}$ получаем выражение

$$
\begin{aligned}
C_{\alpha} \approx & K(0)\left[e_{\alpha, i_{2}, 3,4,5, \ldots, L} J_{2}^{i_{2}}\left(1-\Lambda_{3}\right)\left(1-\Lambda_{4}\right) \ldots\left(1-\Lambda_{L}\right)+\right. \\
& +e_{\alpha, 2, i_{3}, 4,5, \ldots, L}\left(1-\Lambda_{2}\right) J_{3}^{i_{3}}\left(1-\Lambda_{4}\right) \ldots\left(1-\Lambda_{L}\right)+ \\
& \left.+e_{\alpha, 2,3, i_{4}, 5, \ldots, L}\left(1-\Lambda_{2}\right)\left(1-\Lambda_{3}\right) J_{4}^{i_{4}}\left(1-\Lambda_{5}\right) \ldots\left(1-\Lambda_{L}\right)+\cdots\right] .
\end{aligned}
$$

В первом слагаемом в квадратных скобках недиагональный элемент стоит на месте $M_{2 i_{2}}$ (см. формулу (16)). Принимая во внимание явное выражение для $K(0)(17)$, нетрудно видеть, что это слагаемое может быть преобразовано следующим образом:

$$
K(0) e_{\alpha, i_{2}, 3,4,5, \ldots, L} J_{2}^{i_{2}}\left(1-\Lambda_{3}\right)\left(1-\Lambda_{4}\right) \ldots\left(1-\Lambda_{L}\right)=e_{\alpha, i_{2}, 3,4,5, \ldots, L} \frac{J_{2}^{i_{2}}}{1-\Lambda_{2}} .
$$

Это выражение отлично от нуля, только когда $\alpha=1$ или $\alpha=2$. Случай $\alpha=1$ соответствует вычислению поправки к нормировочной константе и интереса для нас не представляет [13]. В случае $\alpha=2$ и $i_{2}=1$ рассматриваемое слагаемое даст коэффициент $C_{2}$, поскольку остальные слагаемые в выражении (18) равны нулю при $\alpha=2$. Аналогичные результаты могут быть получены для всех $\alpha \neq 1$ :

$$
C_{\alpha} \approx-\frac{J_{\alpha}^{1}}{1-\Lambda_{\alpha}}, \quad \alpha \neq 1
$$

\footnotetext{
${ }^{3)}$ Например, если $L=3$, то двум линейно независимым строкам матрицы $M$ можно сопоставить два непараллельных вектора а и b. Тогда решение (15) соответствует нахождению вектора с, ортогонального этим двум. Этот вектор параллелен векторному произведению [a, b], компоненты которого, как известно, могут быть записаны с помощью трехмерного тензора Леви-Чивиты: $c_{i} \sim$ $e_{i j k} a_{j} b_{k}$.
}

5 Теоретическая и математическая физика, т. 171, № 1, 2012 г. 
Как уже было отмечено, нас интересуют только сингулярные по $\omega=U_{2}-U_{1}$ члены разложения (13). Такая сингулярность имеет место только у тех коэффициентов (19), номер которых $\alpha$ соответствует паре индексов с противоположными знаками, т. е. $\alpha \rightarrow n,-n$ [13]. Сингулярность возникает как нуль знаменателя в формуле (19) при $\omega=0$, поэтому энергетические аргументы во всех величинах, кроме знаменателя (19), можно выровнять, положив $U_{1}=U_{2} \equiv U$. При этом величины $J_{\alpha}^{1}$ будут определяться следующими интегралами:

$$
\begin{aligned}
J_{\alpha}^{1} \rightarrow J_{-n n}^{00} & =\int P(\varepsilon) \frac{\mathcal{L}_{U}\left(\varepsilon+x_{1}\right) \mathcal{L}_{U}\left(\varepsilon+x_{2}\right)}{G_{U}^{n}\left(x_{1}\right) G_{U}^{-n}\left(x_{2}\right)} d x_{1} d x_{2} d \varepsilon= \\
& =\int d \varepsilon P(\varepsilon)\left(\frac{\varepsilon^{2}}{\varepsilon^{2}+4-U^{2}}\right)^{|n|} .
\end{aligned}
$$

Интегралы по $x_{1}$ и $x_{2}$, входящие в выражение (20), были вычислены в работе [13]. Таким образом, для сингулярной части первой неисчезающей поправки по беспорядку к функции $\mathcal{R}\left(x_{1} x_{2}\right)$ (обозначим ее $\operatorname{sing} \mathcal{R}\left(x_{1} x_{2}\right)$ ) можно написать следующие выражения:

$$
\begin{aligned}
\operatorname{sing} \mathcal{R}\left(x_{1} x_{2}\right) & =\sum_{n \neq 0} C_{-n n} \sigma_{U}^{n}\left(x_{1}\right) \sigma_{U}^{-n}\left(x_{2}\right)= \\
& =-\sum_{n \neq 0} \frac{J_{-n n}^{00}}{1-\lambda_{n}\left(U_{1}\right) \lambda_{-n}\left(U_{2}\right)} \sigma_{U}^{n}\left(x_{1}\right) \sigma_{U}^{-n}\left(x_{2}\right) .
\end{aligned}
$$

Для малых $\omega$ справедливо разложение (см. формулу (10))

$$
1-\lambda_{n}(U) \lambda_{-n}(U+\omega)=-\frac{2 i n \omega}{\sqrt{4-U^{2}}}+O\left(\omega^{2}\right),
$$

поэтому

$$
\operatorname{sing} R\left(x_{1} x_{2}\right)=\frac{\sqrt{4-U^{2}}}{2 i \omega} \sum_{n \neq 0} J_{-n n}^{00} \frac{\sigma_{U}^{n}\left(x_{1}\right) \sigma_{U}^{-n}\left(x_{2}\right)}{n}
$$

откуда, используя свойство функций (10) $\sigma_{U}^{n}(U-x)=\sigma_{U}^{-n}(x)$, получаем следующее выражение для входящей в формулу (7) функции $\mathcal{F}_{U}\left(x_{1} x_{2}\right)$ :

$$
\mathcal{F}_{U}\left(x_{1} x_{2}\right)=\frac{\sqrt{4-U^{2}}}{2 i} \sum_{n \neq 0} J_{-n n}^{00} \frac{\sigma_{U}^{-n}\left(x_{1}\right) \sigma_{U}^{n}\left(x_{2}\right)}{n} .
$$

С помощью формул (8), (20) и (24) несложно получить, что

$$
\begin{aligned}
W(U)=- & \frac{i}{2} \sqrt{4-U^{2}} \sum_{n \neq 0} \int d \varepsilon \frac{P(\varepsilon)}{n}\left(\frac{\varepsilon^{2}}{\varepsilon^{2}+4-U^{2}}\right)^{|n|} \times \\
& \times \int \sigma_{U}^{n}(x) x d x \lim _{a \rightarrow \infty} \sigma_{U}^{-n}(a) a^{2} .
\end{aligned}
$$

Имея в виду доказанные в работе [13] свойства функций (10)

$$
\int \sigma_{U}^{n}(x) x d x=\frac{i}{2} \frac{n}{|n|} \sqrt{4-U^{2}}, \quad \lim _{a \rightarrow \infty} \sigma_{U}^{-n}(a) a^{2}=\frac{\sqrt{4-U^{2}}}{2 \pi},
$$


а также то, что фигурирующий в выражении (25) ряд легко суммируется с помощью формулы $\sum_{n=1}^{\infty} q^{n} / n=-\ln (1-q)$, получаем для функции $W(U)$ и величины $D$ выражения (4).

В качестве примера рассмотрим модель Ллойда [7] при $p(x)=\left[\pi\left(1+x^{2}\right)\right]^{-1}$. Вычисляя по вычетам интеграл в выражении (4), можно показать, что для этой модели

$$
W(U)=\frac{\left[4-U^{2}\right]^{3 / 2}}{2 \pi} \ln \left(1+\frac{\Delta}{\sqrt{4-U^{2}}}\right) .
$$

В актуальном для нас случае малых $\Delta$ получаем, что $W(U) \approx\left(4-U^{2}\right) \Delta /(2 \pi)$ и $D \approx 16 \Delta /(3 \pi)$. Напомним, что для моделей с конечным вторым моментом $M_{2}$, рассмотренных в работе [13], эти величины всегда пропорциональны $\Delta^{2}$. На рис. 1 приведены зависимость критерия Андерсона $D$ от беспорядка $\Delta$ и спектральная зависимость степени локализации $\left.W(U) d U\right|_{\Delta=0.007}$, полученные для модели Ллойда в компьютерном эксперименте ${ }^{4)}$. Построенные там же соответствующие теоретические зависимости, полученные с помощью формул (4) без какой-либо подгонки, позволяют судить о правильности выполненных в работе расчетов. Из рис. 1а видно, что при малом беспорядке критерий Андерсона $D$ для модели Ллойда действительно линейно зависит от параметра разупорядочения $\Delta$.

\section{3. ЗАКЛЮЧЕНИЕ}

В настоящей работе вычислены величина критерия Андерсона и спектральная зависимость степени локализации для диагонально разупорядоченных $1 D$-моделей, у которых функция распределения энергий узлов $P(\varepsilon)$ не имеет конечных четных моментов, кроме нулевого. Для вычислений производится обобщение предложенной в работе [13] методики построения совместной статистики опережающей и запаздывающей функций Грина на случай случайных систем с расходящимися четными моментами функции $P(\varepsilon)$. Предложенная схема вычислений, опирающаяся на систему специальных функций [15], оказывается более компактной и универсальной, чем описанная в работе [13], и позволяет вычислять критерий Андерсона и спектральную зависимость степени локализации в тех случаях, когда обычная теория возмущений неприменима. В качестве примера рассматривается модель Ллойда [7], для которой получена нетривиальная линейная зависимость критерия Андерсона от степени разупорядочения.

\section{ПРИЛОЖЕНИЕ}

Приведем аргументы, показывающие, что, по крайней мере, в случае слабого беспорядка любое решение уравнения (5) при $\left|U_{1,2}\right|<2$ есть знакопостоянная функция. Из уравнения (5) непосредственно вытекает, что при $x_{1}, x_{2} \rightarrow \pm \infty$ функция $\rho\left(x_{1} x_{2}\right) \approx x_{1}^{-2} x_{2}^{-2} \int d \varepsilon P(\varepsilon) \rho\left(U_{1}-\varepsilon, U_{2}-\varepsilon\right)$ и, следовательно, при больших по модулю $x_{1}$ и $x_{2}$ функция $\rho\left(x_{1} x_{2}\right)$ имеет определенный знак. Убедимся теперь в постоянстве знака функции $\rho\left(x_{1} x_{2}\right)$ в отсутствие беспорядка. Уравнение $(5)$ в этом случае имеет вид

$$
x_{1}^{2} x_{2}^{2} \rho\left(x_{1} x_{2}\right)=\rho\left(U_{1}-\frac{1}{x_{1}}, U_{2}-\frac{1}{x_{2}}\right) .
$$

\footnotetext{
4) Методика компьютерного эксперимента описана в работе [13].
} 

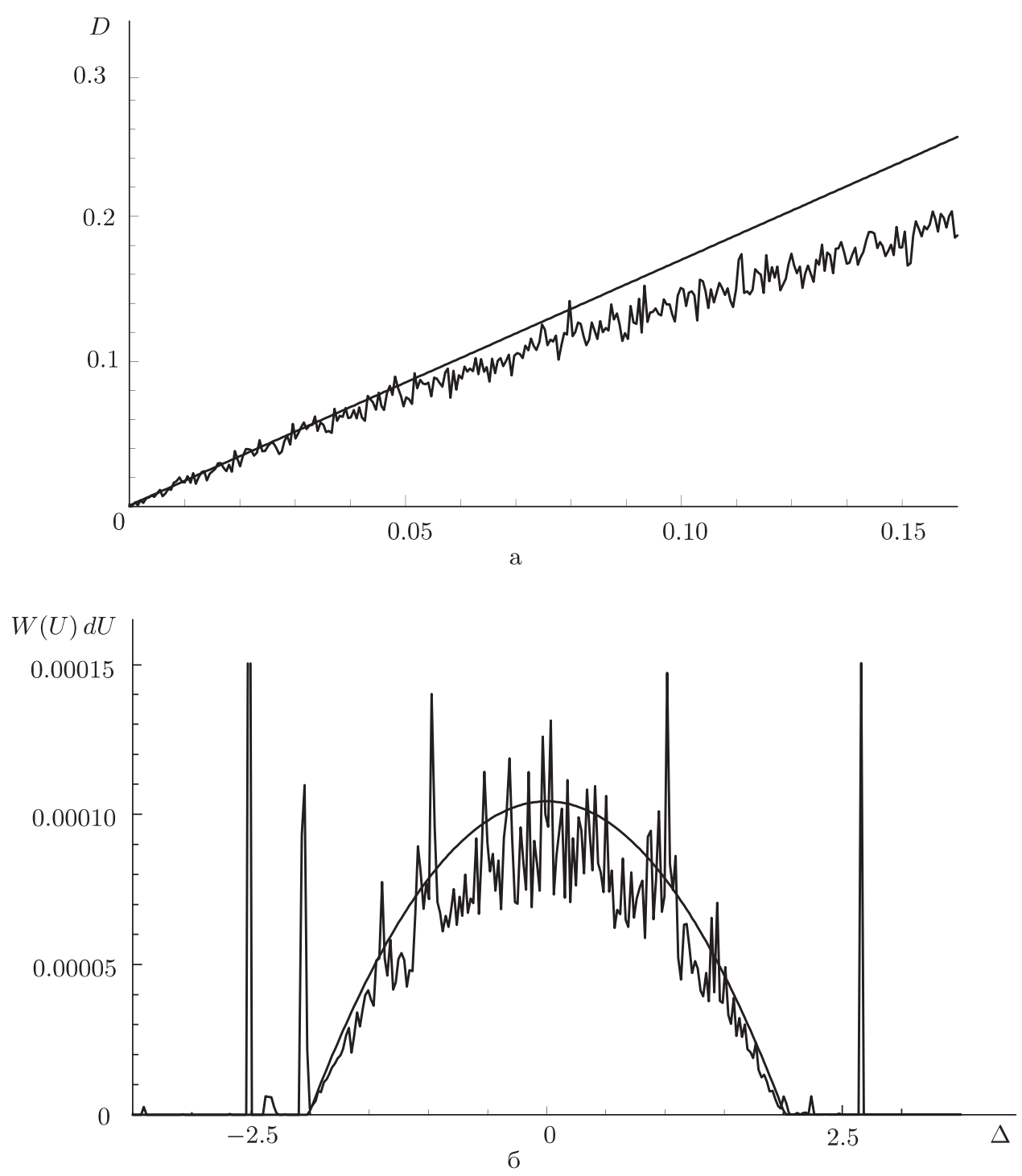

Рис. 1. Зависимость критерия Андерсона $D$ от параметра беспорядка $\Delta$ для модели Ллойда; гладкая линия - расчет по формулам (4), зашумленная линия - компьютерный эксперимент (а). Распределение степени локализации состояний $W(U) d U$ для одномерной модели Ллойда при $\Delta=0.007$ и $d U=7 / 300 ;$ гладкая кривая - расчет по формуле (4), зашумленная кривая компьютерный эксперимент, при котором было выполнено усреднение по 2000 реализаций случайных матриц (1) размером $N=2000$ (б).

Отсюда следует, что на множестве точек с координатами $\left(x_{1, n}, x_{2, n}\right)$ таких, что $x_{i, n+1}=U_{i}-1 / x_{i, n}, i=1,2$, функция $\rho\left(x_{1, n}, x_{2, n}\right)$ сохраняет знак (стартовую точку $\left(x_{1,1}, x_{2,1}\right)$ выберем в области больших по модулю $x_{1}$ и $x_{2}$, где в соответствии со сделанными выше замечаниями постоянство знака имеет место). Процесс построения 


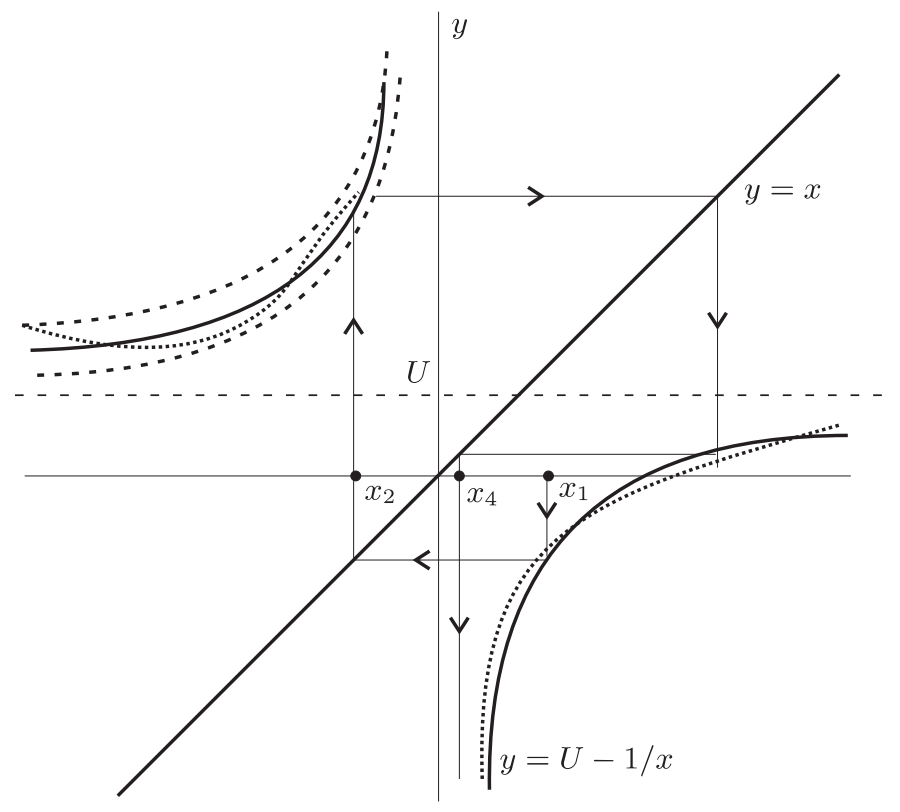

Рис. 2. Процесс построения последовательности точек $x_{n}$.

последовательности точек $x_{n}$ по каждой переменной может быть наглядно представлен стандартным образом с помощью графиков функций $y=U-1 / x$ и $y=x$. На рис. 2 показаны первые несколько шагов этого процесса. Как известно, точки $x_{n}$, получаемые последовательным применением отображения $x_{n+1}=U-1 / x_{n}$, при $|U|<2$ (когда уравнение $x=U-1 / x$ не имеет вещественных решений) заполняют числовую ось с плотностью, определяемой распределением Коши [15], которое нигде не обращается в нуль. Таким образом, при $n \rightarrow \infty$ точки $\left(x_{1, n}, x_{2, n}\right)$ (в которых функция $\rho\left(x_{1} x_{2}\right)$ имеет один и тот же знак) "покрывают" всю плоскость $x_{1}, x_{2}$ и, следовательно, при отсутствии беспорядка функция $\rho\left(x_{1} x_{2}\right)$ знакопостоянна. Неоднозначность выбора стартовой точки дает дополнительные основания для такого заключения.

Аналогичное рассуждение можно провести и в том случае, когда имеет место малый беспорядок. Если функция распределения энергий узлов $P(\varepsilon)$ отлична от нуля только в некотором конечном интервале $[-a, a]$ (т. е. $\int_{-a}^{a} P(\varepsilon) d \varepsilon=1$ ), то для произвольной функции $f(x)$ имеет место соотношение

$$
\int P(\varepsilon) f(x-\varepsilon) d \varepsilon=f(x-\bar{\varepsilon}(x)),
$$

причем функция $\bar{\varepsilon}(x)$ при любом $x$ удовлетворяет условию $\bar{\varepsilon}(x) \in[-a, a]$. Если функция $P(\varepsilon)$ везде отлична от нуля, то соотношение (28) сохраняет силу, однако о функции $\bar{\varepsilon}(x)$ можно сказать лишь, что она ограничена и стремится к нулю при уменьшении беспорядка (т. е. при $\Delta \rightarrow 0$ (3)). Имея это в виду, уравнение (5) может быть переписано в виде

$$
x_{1}^{2} x_{2}^{2} \rho\left(x_{1} x_{2}\right)=\rho\left(U_{1}-\frac{1}{x_{1}}-\bar{\varepsilon}, U_{2}-\frac{1}{x_{2}}-\bar{\varepsilon}\right) \equiv \rho\left(\widetilde{U}_{1}-\frac{1}{x_{1}}, \widetilde{U}_{2}-\frac{1}{x_{2}}\right),
$$


где $\bar{\varepsilon}\left(x_{1} x_{2}\right)$ - ограниченная функция, $\varepsilon_{\min }<\bar{\varepsilon}\left(x_{1} x_{2}\right)<\varepsilon_{\max }$, стремящаяся к нулю при $\Delta \rightarrow 0$. Как видно, уравнение (29) отличается от уравнения (27) только заменой $U_{1,2}$ на $\widetilde{U}_{1,2} \equiv U_{1,2}-\bar{\varepsilon}\left(x_{1} x_{2}\right)$, где функция $\bar{\varepsilon}\left(x_{1} x_{2}\right)$ мала при слабом беспорядке. Произведем теперь графическое построение системы точек $\left(x_{1, n}, x_{2, n}\right)$, в которых функция $\rho\left(x_{1} x_{2}\right)$ сохраняет знак, аналогичное описанному выше для случая нулевого беспорядка. При этом следует заменить гиперболическую функцию $U-1 / x$ на функцию $U-\bar{\varepsilon}(x, z)-1 / x$, и при построении $x_{1, n+1}$ следует положить $x=x_{1, n}$, $z=x_{2, n}$, а при построении $x_{2, n+1}-x=x_{2, n}, z=x_{1, n}$. Схематически такая замена показана на рис. 2 пунктиром. При малом беспорядке указанная замена не может качественно изменить картину получения множества точек, в которых функция $\rho$ имеет один и тот же знак, поскольку для предельных функций $U_{1,2}-\varepsilon_{\min }-1 / x$ и $U_{1,2}-\varepsilon_{\max }-1 / x$ (показанных на рис. 2 пунктиром во втором квадранте) построение ничем не отличается от описанного выше для случая $\Delta=0$. Качественное изменение было бы возможно, например, при появлении точек пересечения графиков функций $y=\widetilde{U}(x)-1 / x$ и $y=x$. Однако при малом беспорядке (и, следовательно, при малой функции $\left.\bar{\varepsilon}\left(x_{1} x_{2}\right)\right)$ такая возможность может возникнуть лишь при условии, что энергетические параметры $\left|U_{1,2}\right|$ находятся в малой области вблизи 2 , размер которой стремится к нулю при $\Delta \rightarrow 0$. Для выполненных в настоящей работе расчетов первого неисчезающего приближения для спектральной зависимости локализации $W(U)$ это несущественно.

\section{Список литературы}

[1] E. E. Jelley, Nature, 38:3502 (1936), 1009-1010.

[2] G. Scheibe, Angew. Chem., 50:11 (1937), 212-219.

[3] И. М. Лифшиц, С.А. Гредескул, Л.А. Пастур, Введение в теорию неупорядоченных систем, Наука, М., 1982.

[4] A. Rodríguez, V.A. Malyshev, G. Sierra, M. A. Martín-Delgado, J. Rodríguez-Laguna, F. Domínguez-Adame, Phys. Rev. Lett., 90:2 (2003), 027404, 4 pp.

[5] V.A. Malyshev, A. Rodrígues, F. Domíngues-Adame, Phys. Rev. B, 60:20 (1999), 14140-14146, arXiv: cond-mat/9908438.

[6] M. Titov, H. Schomerus, Phys. Rev. Lett., 91:17 (2003), 176601, 4 pp., arXiv: cond-mat/0302580.

[7] P. Lloyd, J. Phys. C, 2:10 (1969), 1717-1725.

[8] D. J. Thouless, J. Phys. C, 16:26 (1983), L929.

[9] A. MacKinnon, J. Phys. C, 17:10 (1984), L289-L292.

[10] D. E. Rodrigues, H. M. Pastawski, J. F. Weisz, Phys. Rev. B, 34:12 (1986), 8545-8549.

[11] S. Fishman, R. E. Prange, M. Griniasty, Phys. Rev. A, 39:4 (1989), 1628-1633.

[12] Lev I. Deych, A. A. Lisyansky, B. L. Altshuler, Phys. Rev. Lett., 84:12 (2000), 2678-2681.

[13] Г. Г. Козлов, ТМФ, 162:2 (2010), 285-303.

[14] P. W. Anderson, Phys. Rev., 109:5 (1958), 1492-1505.

[15] G. G. Kozlov, Spectrum and eigen functions of the operator $H_{U} f(x) \equiv f(U-1 / x) / x^{2}$ and strange attractor's density for the mapping $x_{n+1}=1 /\left(U-x_{n}\right)$, arXiv: 0803.1920. 